\title{
Aplicación del branding emocional en el marketing del sector funerario
}

\section{Emocional branding aplication in the funeral sector marketing}

\author{
Danna Paola Álvarez Pérez ${ }^{1}$, Carlos Alberto Pacheco Sánchez ${ }^{2}$ \\ ${ }^{1}$ Estudiante, Administración de Empresas, Universidad Francisco de Paula Santander Ocaña, Colombia \\ ORCID: https://orcid.org/0000-0003-4539-2821, E-mail: dpalvarezp@ufpso.edu.co \\ ${ }_{2}^{2}$ Magister, Administrador de Empresas, Universidad Francisco de Paula Santander Ocaña, Colombia \\ ORCID: https://orcid.org/0000-0002-9369-582X, E-mail: capachecos@ufpso.edu.co
}

Cómo citar: Álvarez - Pérez, D. P., \& Pacheco - Sánchez, C. A. (2020). Aplicación del branding emocional en el marketing del sector funerario. Revista Científica Profundidad Construyendo Futuro, 8(8), 16-21. https://doi.org/10.22463/24221783.2618

Recibido: 15 de Enero de 2017 / Aprobado: 22 de Febrero de 2018

\begin{abstract}
Resumen
Mantener una buena cultura emprendedora en la educación permite generar un espíritu empresarial sustentable, de allí que, su aplicabilidad permite el diseño de acciones estratégicas que impulsan e inspiran al alumno a edificar su idea empresarial, por consiguiente, el objetivo del estudio fue identificar las habilidades y capacidades emprendedoras que poseen los estudiantes de Administración de Empresas, de igual forma, la incidencia que tienen las asignaturas del programa. En esta medida, la investigación empleó una metodología cuantitativa con un enfoque descriptivo, dado que esta modalidad brinda la posibilidad de realizar un análisis más profundo de cada una de las características de los individuos; se aplicó como técnica de recolección la encuesta a 144 estudiantes de diferentes semestres, donde se concluye que la población estudiantil poseen grandes capacidades y habilidades para emprender, sin embargo, la educación que es impartida no influye lo suficiente para que edifiquen empresas que contribuyan al desarrollo regional.
\end{abstract}

Palabras claves: Cultura, Emprendimiento, Estudiantes, Espíritu emprendedor, Habilidades, Universidad.

\begin{abstract}
: Maintaining a good entrepreneurial culture in education allows to generate a sustainable entrepreneurial spirit, hence, its applicability allows the design of strategic actions that drive and inspire students to build their business idea, therefore, the objective of the study was to identify the entrepreneurial skills and abilities that students of Business Administration possess, likewise, the impact that the program's subjects have. In this measure, the research used a quantitative methodology with a descriptive approach, since this modality offers the possibility of making a deeper analysis of each of the characteristics of the individuals; the survey was applied as a collection technique to 144 students from different semesters, where it is concluded that the student population has great capacities and abilities to undertake, however, the education that is given does not influence enough for them to build enterprises that contribute to regional development.
\end{abstract}

Key words: Culture, Entrepreneurship, Students, Entrepreneurship, Skills, University. 


\section{Introducción}

En la actualidad, existe una gran cantidad de técnicas y estrategias para dar a conocer una marca, desde luego, el uso de las mismas se convierte en un fenómeno global, tal como se relaciona en los últimos años el branding emocional.

Cabe resaltar, que no todas logran fomentar el deseo de formar una necesidad humana en el consumidor. Esto se debe a que muchas empresas, principalmente las pequeñas organizaciones, pasan por alto el proceso de Branding, ya que posiblemente por su poca experiencia, se consideran a sí mismas como un negocio y no como una marca.

Para abordar un concepto claro de Marketing, se hace referencia a una función organizacional y/o un conjunto de procesos para crear, comunicar y agregar valor a los clientes con el fin de administrar las relaciones con los mismos de forma que beneficien a la organización y a todos sus grupos de interés. (Kotler, 2012)

Por otro lado, Smith (2012) especialista en Branding y gestión estratégica de marca, define dicho término como todo aquello que hace conectar la estrategia de la marca con la experiencia del consumidor para que, de esa manera, tener como consecuencia la lealtad y la preferencia del mismo. Es por ello que la aplicación de Branding en las empresas es de suma importancia, ya que estos procesos permiten formar un vínculo muy significativo de parte del cliente con la compañía gracias al desarrollo de esta técnica.

Es así, como es necesario hacer utilización de estrategias de Branding para lograr el éxito, ya que con este se obtiene "enamorar" al consumidor de la marca. De otra manera, según Porter (1991) los clientes son una de las fuerzas del entorno que influyen en la competitividad de la empresa mediante sus expectativas y requerimientos. Es el consumidor quien tiene la potestad, en cierta parte, de influir en la competitividad de las organizaciones. Por lo que surge la incógnita sobre cómo en el sector funerario de Ocaña, desarrolla estrategias para manejar altos estándares de procesos con el fin de construir técnicas innovadoras en pro de una mejora e incrementar en los clientes el afecto hacia la marca.

Es curioso cómo en la industria funeraria es posible relacionar estrategias de mercado y el manejo de la emoción para la fidelización del cliente, teniendo en cuenta que el adaptarse al manejo del dolor a causa de la pérdida de seres queridos y las emociones que este tipo de situaciones genera, es interesante, pero sobre todo complejo, ya que manejar las emociones de las demás personas resulta un tanto intrincado. Dadas las circunstancias, el proyecto que se ejecuta, tiene el fin de conocer la aplicación del branding emocional en el marketing, logrando este objetivo a través de diagnóstico donde se analizan las acciones y estrategias del sector funerario de Ocaña, para despertar una emoción provocada por los sentimientos, la imaginación, las historias y los momentos vividos del consumidor, a consecuencia de alcanzar el éxito de una marca estableciendo vínculos y asegurando lealtad y fidelidad del mismo, siendo la empresa, un generador de necesidad humana. 


\section{Marco Teórico}

Porter (2008) en su libro de las cinco fuerzas competitivas que le dan forma a la estrategia, revela que hay cinco fuerzas que dan forma a la competencia. La competencia y en efecto la rivalidad de las empresas es algo inevitable ya que cada día vemos cómo crece el mercado, vemos el lanzamiento de nuevos productos y la implementación de nuevas estrategias por parte de las mismas. Es por eso que se ve la necesidad de defender a las empresas de las fuerzas competitivas y moldearlas para su propio beneficio es crucial para la estrategia.

A nosotros como seres humanos nos mueve la emoción que nuestro entorno proporcione, es por eso que a la hora de aplicar Branding emocional es vital mencionar la teoría de Goleman (1996), en ella se plantea, que más allá de pensar en que la inteligencia es un aspecto cognitivo avanzado que depende de la buena memoria y buenas capacidades para resolver problemas, de igual forma, la inteligencia emocional es nuestra conciencia, es la capacidad de reconocer emociones, pero sobre todo de dirigirnos a los demás cómo manejar los impulsos, el autocontrol, la empatía y la motivación.

Finalmente, se puede deducir que es inevitable no moverse a la emoción que causa nuestro entorno externo ya que como seres humanos tenemos esas capacidades innatas.

\section{Metodología}

La investigación fue de tipo cuantitativo ya que sustentado por Hernández (2006) señala que este tipo de investigación tiene como fin la búsqueda de datos estadísticos que fundamenten la realidad objetiva a partir de mediciones y agregado a eso es un proceso inductivo que establece un enfoque subjetivo el cual busca información e ideas a interpretar para enriquecer la investigación.

Posteriormente, la investigación es de tipo no probabilística, teniendo en cuenta que, según Hernández Sampieri, Fernández Collado y Baptista Lucio, Metodología de la investigación (2006) la investigación con muestra no probabilística se basa en que la elección de los elementos no depende de la probabilidad sino de las causas relacionadas con las características de la investigación o de quien hace la muestra. Siendo esta la metodología a utilizar, seleccionando 5 funerarias a encuestar y en los que se tuvieron criterios como: Funerarias que estuvieron dispuestas a dar información interna sobre sus procesos, fueron seleccionadas las funerarias que tienen cierto reconocimiento en el municipio, fueron seleccionadas las funerarias que están ubicadas en Ocaña.

Según Gross (2010) la investigación descriptiva se basa en un diagnóstico, ya que buena parte de lo que se escribe y se estudia sobre lo social no va mucho más allá de este nivel, siendo este proyecto una investigación dada a describir el sector funerario. Como técnica se hace uso de la encuesta, proporcionando 17 preguntas con las cuales después de aplicarlas se procede al análisis de estadísticas.

\section{Resultados}

El estudio presenta los siguientes hallazgos: 
Tabla 1. Conocimiento sobre el Marketing

\begin{tabular}{lll}
\hline & Frecuencia & Porcentaje \\
\hline Sí & 4 & $80 \%$ \\
No & 1 & $20 \%$ \\
Total & 5 & $100 \%$ \\
\hline
\end{tabular}

Nota: La tabla se puede observar la cantidad de funerarias que conocen y que desconocen el Marketing y posteriormente los porcentajes correspondientes. Fuente: encuesta aplicada por el autor del proyecto.

En el mundo empresarial y en todo lo relacionado con el mercado, el Marketing juega un papel considerablemente importante ya que gracias a este proceso se establece la comunicación e información entre compañías y clientes. Sin embargo, en las funerarias de Ocaña, las estadísticas afirman que solo el $80 \%$ de dichas empresas tienen conocimiento de los procesos de Marketing y que, en efecto, el $20 \%$ restante lo desconoce.

Tabla 2. Aplicación de Marketing

\begin{tabular}{lll}
\hline $\begin{array}{l}\text { Aplicación de } \\
\text { Marketing }\end{array}$ & Frecuencia & Porcentaje \\
\hline Sí & 5 & $100 \%$ \\
No & 0 & $0 \%$ \\
Total & 5 & $100 \%$ \\
\hline
\end{tabular}

Nota: En esta tabla se puede evidenciar la cantidad de funerarias que aplican Marketing en sus procesos. Fuente: Elaboración propia.

Partiendo del referente de Nieves (2018) "el Marketing es un conjunto de técnicas que son vitales para generar y conservar la confianza del cliente", por ello, se considera que la aplicación de dicho proceso es indispensable en todas y cada una de las empresas.
Tabla 3. Conocimiento sobre Branding emocional

\begin{tabular}{lcc}
\hline & Frecuencia & Porcentaje \\
\hline Sí & 1 & $20 \%$ \\
No & 4 & $80 \%$ \\
Total & 5 & $100 \%$ \\
\hline
\end{tabular}

Nota: en esta tabla se puede observar la cantidad de funerarias que tienen conocimiento sobre Branding emocional. Fuente: Elaboración propia.

El Branding emocional es aquello que hace diferencias una de la otra, teniendo en cuenta que la finalidad del mismo es conectar la marca con las emociones del cliente y usuario. Así mismo, se ve la necesidad de aplicar dicho proceso en las funerarias de Ocaña ya que es de suma importancia, sin embargo, solo el $20 \%$ de las funerarias tiene conocimiento acerca del Branding emocional $\mathrm{y}$, en consecuencia, el $80 \%$ lo desconoce.

Tabla 4. Empatía con el cliente

\begin{tabular}{lll}
\hline & Frecuencia & Porcentaje \\
\hline Sí & 5 & $100 \%$ \\
No & 0 & $0 \%$ \\
Total & 5 & $100 \%$ \\
\hline
\end{tabular}

Nota: en esta tabla se dan a conocer la cantidad de las funerarias que en el momento del duelo se empatizan con el cliente. Fuente: Elaboración propia.

De acuerdo a la teoría de las emociones propuesta por Goleman (1996) señala que la empatía es una de las habilidades que toda persona debe tener para con sus clientes. De esa manera, se puede deducir que, según las estadísticas, el $100 \%$ de las funerarias de Ocaña tiene una alta empatía con el cliente, es decir, cumplen con una de las variables del Branding. 
Tabla 5. Comunicación frecuente con clientes potenciales

\begin{tabular}{lll}
\hline & Frecuencia & Porcentaje \\
\hline Sí & 5 & $100 \%$ \\
No & 0 & $0 \%$ \\
Total & 5 & $100 \%$ \\
\hline
\end{tabular}

Nota: en esta tabla se puede observar la cantidad de funerarias que mantienen una comunicación frecuente con los clientes potenciales. Fuente: encuesta aplicada por el autor del proyecto.

La comunicación frecuente con el cliente potencial tiene consecuencias como la fidelización del mismo, es por eso que dicho proceso es tan importante en todas y cada una de las empresas y en este caso, en las funerarias. Este tipo de comunicación con clientes potenciales conlleva a que posean la convicción de que escoger una empresa entre tantas sea su mejor opción. En las funerarias de Ocaña, según las estadísticas la totalidad de las mismas mantienen una comunicación frecuente con los clientes potenciales, teniéndolos en cuenta a la hora de desarrollar actividades de las cuales se pueden beneficiar los clientes.

Tabla 6. Honestidad

\begin{tabular}{lll}
\hline Honestidad & Frecuencia & Porcentaje \\
\hline Sí & 5 & $100 \%$ \\
No & 0 & $0 \%$ \\
Total & 5 & $100 \%$ \\
\hline
\end{tabular}

Nota: en esta tabla se dan a conocer la cantidad de las funerarias que al momento de exponer sus planes integrales son honestos con el cliente. Fuente: encuesta aplicada por el autor del proyecto.

La honestidad a la hora de ofrecer o de exponer los planes integrales de las funerarias es un componente muy importante, ya que de alguna u otra manera ese es uno de los valores o de las habilidades que llevan a seducir a un cliente. En este caso, según Sócrates y citado por Diario concepción (2016) "la honestidad es un valor o cualidad propia de los seres humanos, relacionado con los principios de verdad y justicia y con la integridad moral". Con la anterior referencia y basándose en las estadísticas, el $100 \%$ de las funerarias de Ocaña son honestos a la hora de exponer sus planes integrales al cliente.

Tabla 7. Asesoría comercial puerta a puerta

\begin{tabular}{|c|c|c|}
\hline $\begin{array}{l}\text { Asesoría } \\
\text { comercial } \\
\text { puerta } \\
\text { puerta }\end{array}$ & Frecuencia & Porcentaje \\
\hline Sí & 4 & $80 \%$ \\
\hline No & 1 & $20 \%$ \\
\hline Total & 5 & $100 \%$ \\
\hline
\end{tabular}

Nota: en esta tabla se puede observar la cantidad de funerarias de Ocaña que manejan asesoría puerta a puerta. Fuente: encuesta aplicada por el autor del proyecto.

Para esclarecer un poco sobre la asesoría puerta a puerta, son aquellas visitas que los asesores comerciales de las empresas llevan a cabo en una población específica con el fin de que la personas tengan conocimiento sobre los planes que manejan $\mathrm{u}$ ofertando ciertos paquetes. La asesoría puerta a puerta es una manera de hacer Marketing y Branding debido a que de esa manera se está llegando al cliente sin necesidad de que este tenga que dirigirse a las instalaciones, es por ello que para algunas empresas resulta siendo esta una estrategia esencial. Las estadísticas afirman que el $80 \%$ de las funerarias en Ocaña aplican en sus procesos la asesoría puerta a puerta y que el $20 \%$ restante no maneja dicho proceso.

\section{Conclusiones}

La investigación permite evidenciar que las empresas que conforman el sector 
funerario de Ocaña conocen el Marketing y que, en consecuencia, lo aplican, pero no tienen conocimiento amplio sobre el Branding emocional (80\%), lo que lleva a deducir que las funerarias deben centrar los fines corporativos no solo en asegurar las ventas y prestar los servicios, sino también, generar escenarios inspiradores $\mathrm{y}$ motivacionales.

Por otro lado, la estrategia de puerta a puerta, el enfoque de la empatía y la honestidad que se aplica por más del $60 \%$ de las empresas fúnebre, son necesarias de mantener pensando en el desarrollo de las ventas y en la construcción de branding emocional más cuando se trata de la entrega de servicios a clientes que presentan un comportamiento desmotivacional y melancólico.

Finalmente, las empresas hacen un acompañamiento permanente a los afiliados mediante profesionales expertos que orientan la comunicación y el proceso de adquisición de los planes integrales referenciados en su portafolio de servicios.

\section{Referencias}

Diario concepción. (27 de Julio de 2016). Obtenido de https://www.diarioconcepcion.cl/edit orial/2016/07/27/la-honestidadsegun-socrates.html

Goleman , D. (1996). Inteligencia emocional . Kairós.

Gross, M. (2010).

Hernández Sampieri , R. (2006). Metodología de la investigación. Cuarta edición . Mc Graw Hill.
Hernández Sampieri, R., Fernández Collado , J., \& Baptista Lucio , P. (2006). En Metodología de la investigación. México: Mc Graw Hill.

Kotler, P. (2012). Dirección de Marketing. México: Pearson.

Nieves , G. (26 de Noviembre de 2018). Obtenido de https://www.postedin.com/blog/porque-el-marketing-en-las-empresases-tan-importante/

Porter, M. (1991). La ventaja competitiva de las naciones. Buenos Aires: Vergara.

Porter , M. (2008). Las cinco fuerzas competitivas que le dan fuerza a la estrategia.

Smith, C. (08 de Mayo de 2012 ). PuroMarketing. Obtenido de https://www.puromarketing.com/98/1 3002/frase-branding.html 